\title{
ON ANALYTIC MAPS OF PLANE DOMAINS
}

\author{
By James A. Jenkins and Nobuyuki Suita
}

Dedicated to Professor Kôtaro Oikawa on his 60 th birthday

\section{Introduction.}

Let $D$ be a finitely connected plane domain, having more than two nondegenerate boundary components. We may assume that $D$ is bounded by analytic curves.

An analytic map $f(z)$ of $D$ is called boundary preserving, if every sequence $\left\{z_{\nu}\right\}_{\nu=1}^{\infty}$ of $D$ tending to the boundary $\partial D$ is mapped onto a sequence $\left\{f\left(z_{\nu}\right)\right\}_{\nu=1}^{\infty}$ tending to the boundary of the image domain $\Delta=f(D)$. A boundary preserving map $f(z)$ of $D$ covers the image domain $\Delta$ finitely many times, $N$. By making use of circular slit mappings, we shall show a uniqueness theorem of an analytic map $f(z)$.

A boundary preserving map $f(z)$ is extended over the doubled surface $\hat{D}$ and the extended map $\hat{f}(z)$ is an analytic map of the closed Riemann surface $\hat{D}$ onto the doubled surface $\hat{\Delta}$. The Seveli-deFranchis' Theorem [3] states that the number of analytic map from a compact Riemann surface $\hat{D}$ of genus greater than one into a compact Riemann surface $\hat{\Delta}$ of genus greater than one is finite. Recently A. Howard and A. Sommes [2] gave a bound of the number of analytic maps of a compact Reimann surface of genus $g \geqq 2$, which was

$$
(2 \sqrt{6}(g-1)+1)^{2+2 g^{2}} g^{2}(g-1) \sqrt{2^{8(g-1)}}+84(g-1) .
$$

If the connectivity of $D$ in $n \geqq 3$, the genus of $D$ is equal to $n-1$. As an application of the uniqueness theorem we obtain a simple bound of the number of boundary preserving maps of $D$ into domain of connectivity $\geqq 3$

$$
(n-2) 2^{4 n-6} \text {. }
$$

\section{Uniqueness problem.}

Let $D$ be an $n$-ply connected plane domain bounded by $n$ analytic curves. Let $f(z)$ be an boundary preserving analytic map of $D$ onto an $m$-ply connected

Research supported in Part by the National Science Foundation.

Received September 2, 1987 
domain $\Delta$, with $m \geqq 2$. Suppose that $f(z)$ covers $\Delta N$ times. The number $N$ is called the degree of $f$. For two boundary components $\alpha_{1}, \alpha_{2}$ of $\Delta$, let $\left\{C_{\nu}^{(1)}\right\}_{\nu=1}^{N}$ and $\left\{C_{\nu}^{(2)}\right\}_{\nu=1}^{N}$ be their inverse images under $f(z)$ respectively. If a boundary component $C$ covers $\alpha_{1} k$ times $(k \leqq N)$, we write $C k$ times in $\left\{C_{\nu}^{(1)}\right\}_{\nu=1}^{N}$. We prove

THEOREM 1. Let $f(z)$ and $g(z)$ be two boundary preserving analytic maps whose image domains are $\Delta$ and $\Omega$ respectively. If they have the same inverse images $\left\{C_{\nu}^{(1)}\right\}_{\nu=1}^{N}$ and $\left\{C_{\nu}^{(2)}\right\}_{\nu=1}^{N}$, then there exists a conformal mapping $\phi$ of $\Omega$ onto $\Delta$ such that $f(z)=\phi(g(z))$.

Proof. The analytic maps $f(z)$ and $g(z)$ carry $\left\{C_{\nu}^{(j)}\right\}_{j=1}^{N}$ onto boundary components $\alpha$, of $\Delta$ and $\beta$, of $\Omega$ respectively $(j=1,2)$. There exists circular slit annulus mappings $\Phi_{\Delta}$ and $\Phi_{\Omega}$ which map $\alpha_{1}$ and $\beta_{1}$ onto the unit circles, $\alpha_{2}$ and $\beta_{2}$ onto two circles with radii greater than 1 , and the other boundary components (if any) onto circular slits centered at the origin.

We consider a module problem for the family $\Gamma$ of cycles $\{r\}$ which separates $\left\{C_{\nu}^{(1)}\right\}_{\nu=1}^{N}$ and $\left\{C_{\nu}^{(2)}\right\}_{\nu=1}^{N}$. Then two metrics

and

$$
\frac{1}{2 \pi N}\left|\frac{d \Phi_{\Delta}}{\Phi_{\Delta}}\right|=\frac{\left|\Phi_{\Delta}^{\prime}(f(z))\right|\left|f^{\prime}(z)\right|}{2 \pi N\left|\Phi_{\Delta}(f(z))\right|}|d z|
$$

$$
\frac{1}{2 \pi N}\left|\frac{d \Phi_{\Omega}}{\Phi_{\Omega}}\right|=\frac{\left|\Phi_{\Omega}^{\prime}(g(z))\right|\left|g^{\prime}(z)\right|}{2 \pi N\left|\Phi_{\Omega}(g(z))\right|}|d z|
$$

defines the extremal metrics. Since the extremal metric is unique $[4,5]$, we have

$$
\frac{\Phi_{\Delta}^{\prime}(f(z))}{\Phi_{\Delta}(f(z))} f^{\prime}(z)=e^{\imath \lambda} \frac{\Phi_{\Omega}^{\prime}(g(z))}{\Phi_{\Omega}(g(z))} g^{\prime}(z)
$$

By integration, we deduce that $e^{\imath \lambda}=1$ and $\Phi_{\Delta}(f(z))=e^{i \theta} \Phi_{\Omega}(g(z))$. Thus we obtain

with

$$
f(z)=\phi(g(z)) \text {, }
$$

$$
\phi=\Phi_{\Delta}^{-1} \circ e^{i \theta} \Phi_{\Omega}
$$

Remark. The finiteness of connectivity of $D$ is inessential, if the boundary components $\alpha_{1}$ and $\alpha_{2}$ are both nonweak [4]. In this case the uniqueness of the extremal metrics is true $[4,5]$ and Theorem 1 is valid.

When $\Omega$ coincides with $\Delta$, we obtain

COROLLARY. Among boundary preserving analytic maps of $D$ onto $\Delta$ with both connectivities $\geqq 3$, a distribution of the inverse images of three boundary components of $\Delta$ determines a unique analytic map.

Proof. Let $\alpha_{1}, \alpha_{2}$ and $\alpha_{3}$ be three boundary components of $\Delta$. By making use of a circular slit annulus mapping $\Phi_{\Delta}(w)$ of $\Delta$, which carries $\alpha_{1}$ and $\alpha_{2}$ onto the circles $\left|\Phi_{\Delta}\right|=1$ and $\left|\Phi_{\Delta}\right|=R>1$ respectively, we have 


$$
\Phi_{\Delta}(f(z))=e^{i \theta} \Phi_{\Delta}(g(z)) .
$$

The third boundary component $\alpha_{3}$ is carried onto a circular slit. We have $e^{i \theta}=1$ and $f(z)=g(z)$.

\section{The number of analytic maps.}

In this section we assume that the connectivity $n$ of a domain $D$ is greater than two. Let $f(z)$ be a boundary preserving map of $D$ onto $\Delta$ whose connectivity $m$ is greater than two.

A boundary preserving map $g(z)$ of $D$ is said to be equivalent to $f(z)$ if there exists a conformal mapping $\phi$ of the image domain $\Omega$ under $g$, which is not a non-identity selfconformal mapping of $\Delta$ and satisfies $f(z)=\phi(g(z))$. We estimate the number of equivalence classes of all boundary preserving maps $f(z)$.

We prove

THEOREM 2. The number of equivalence classes of boundary preserving analytic maps of an n-ply connected domain $D$ is at most $(n-2) 2^{4 n-6}$.

Proof. Let $f(z)$ be a boundary preserving map of degree $N$. Since $f$ is boundary preserving, $f$ has no branch points on the boundary. The total order of branch points in $D$ is denoted by $V$. Then the Riemann-Hurwitz relation gives

$$
n-2=N(m-2)+V,
$$

$m$ being the connectivity of $\Delta \geqq 3$. Then we have

$$
1 \leqq N \leqq n-2 \text {. }
$$

We may assume that $\Delta$ is a circular slit annulus such that $\alpha_{1}$ and $\alpha_{2}$ are the unit circle and a circle with radius greater than 1 . We deduce from Theorem 1 and its corollary that the number of choices of inverse images of $\alpha_{1}$ and $\alpha_{2}$ is bounded by ${ }_{n} H_{N}^{2}$ where ${ }_{n} H_{N}$ means the multiple combination. After the inverse images of $\alpha_{1}$ and $\alpha_{2}$ are determined, there remain $m-2$ possibilities of rotations, since the number of circular slits is equal to $m-2$. Thus the total number of such maps is dominated by

$$
\begin{aligned}
\sum_{N=1}^{n-2}(m-2)_{n} H_{N}^{2} & \leqq(n-2) \sum_{N=1}^{n-2} n+N-1 C_{N}^{2} \\
& \leqq(n-2) \sum_{N=1}^{n-2} 2 n-3 \\
& \leqq(n-2) 2^{2} \leqq(n-2)\left(\sum_{N=0}^{2 n-6} 2 n-3\right.
\end{aligned}
$$




\section{Special cases.}

We denote by $A_{n}$ the maximal number of the equivalence classes of boundary preserving analytic maps of $n$-ply connected domains $D$, bounded by $n$ analytic curves. In the case $n=3$, the Riemann-Hurwitz relation shows that $N=1$. The maximal number of conformal self mappings was determined by Heins [1]. From his result we have $A_{3}=6$.

In the case $n=4$, the Riemann-Hurwitz relation is

$$
2=N(m-2)+V
$$

Then possible solutions are $V=0, N=2, m=3, V=0, N=1, m=4$ and $V=1, N=1$, $m=3$ which does not occur.

The maximal number of conformal self-mapping of domains of connectivity 4 is equal to 12 and in this case each conformal self mapping other than identity fixes one boundary component and permutes the other three boundary components [1]. We show that there are no boundary preserving maps of degree two on such a domain $D_{12}$. In fact, if there were a boundary preserving map $f$ of degree two, the image domain would be a triply connected, and one boundary component is covered by two boundary components of $D_{12}$ and the other two are covered by the remained two boundary components twice. Hence interchange of the two sheets of the Riemanian image $f\left(D_{12}\right)$ would induce a conformal mapping which fixes two boundary components.

Next large order of conformal automorphism of a domain of connectivity 4 is 8 since any order divides the order of symmetric group $S_{4}$. Such a domain is conformally represented as a domain $D_{8}$ whose boundary components are four circular arcs on the unit circle, whose length are all $2 h<\pi / 4$ and whose midpoints are $1, \imath,-1,-i$. The conformal automorphism of $D_{8}$ is generated $\imath z$ and $1 / z$. The analytic map

$$
f(z)=-\left(\frac{z-1}{z+1}\right)^{2}
$$

is boundary preserving and of degree 2 . The boundary components of the image domain are segments $\left[0, \tan ^{2} h\right],\left[\tan ^{2}(\pi / 4-h), \tan ^{2}(\pi / 4+h)\right]$ and $\left[\cot ^{2} h, \infty\right]$. $f(z)$ is invariant under $1 / z$ and $f_{\nu}(z)=f\left(i^{\nu} z\right), \nu=0,1,2,3$ yield four boundary preserving analytic maps. In order to show that those four are the whole boundary preserving maps of degree 2, we investigate self conformal maps of the image domain $\Delta$. Clearly $1 / w$ is a self conformal map of $\Delta$ and we have

$$
1 / f_{\nu}(z)=f_{\nu+2}(z) \quad(\bmod 4) .
$$

If $\Delta$ has another self conformal map, it has six conformal maps. Then $\Delta$ is conformally equivalent to a circular slit plane with the boundary components

$$
C_{\jmath}=\left\{z \mid z=e^{i \theta}, \frac{2 j}{3} \pi-2 k<\theta_{j}<\frac{2 j}{3} \pi\right\},
$$




$$
j=0,1,2, \quad 0<k<\frac{\pi}{6} .
$$

The linear transformation

$$
-\frac{\left(1+i \tan \left(\frac{\pi}{3}+k\right)\right) z-\left(1-\imath \tan \left(\frac{\pi}{3}+k\right)\right)}{\left(1-i \tan \left(\frac{\pi}{3}+k\right)\right) z-\left(1+\imath \tan \left(\frac{\pi}{3}+k\right)\right)}
$$

maps the circular slit domain onto a parallel slit domain with the slits

and

$$
\begin{aligned}
& {\left[0, \frac{\tan \left(\frac{\pi}{3}+k\right)-\tan \left(\frac{\pi}{3}-k\right)}{\tan \left(\frac{\pi}{3}+k\right)+\tan \left(\frac{\pi}{3}-k\right)}\right],} \\
& {\left[\frac{\tan \left(\frac{\pi}{3}+k\right)-\tan k}{\tan \left(\frac{\pi}{3}+k\right)+\tan k}, \frac{\tan \left(\frac{\pi}{3}+k\right)+\tan k}{\tan \left(\frac{\pi}{3}+k\right)-\tan k}\right]}
\end{aligned}
$$

$$
\left[\frac{\tan \left(\frac{\pi}{3}+k\right)+\tan \left(\frac{\pi}{3}-k\right)}{\tan \left(\frac{\pi}{3}+k\right)-\tan \left(\frac{\pi}{3}-k\right)}, \infty\right] .
$$

If the image domain $\Delta$ has six self conformal maps, we have the following equations.

$$
\left\{\begin{array}{l}
\tan ^{2} h=\frac{\sin 2 k}{\sin \frac{2}{3} \pi} \\
\tan ^{2}\left(\frac{\pi}{4}-h\right)=\frac{\sin \frac{\pi}{3}}{\sin \left(\frac{\pi}{3}+2 k\right)}
\end{array}\right.
$$

which is equivalent to

$$
\left\{\begin{array}{l}
\sin 2 k=\frac{\sqrt{3}}{2} \tan ^{2} h \\
\sin \left(\frac{\pi}{3}+2 k\right)=\frac{\sqrt{3}}{2}\left(\frac{1+\tan h}{1-\tan h}\right)^{2}
\end{array}\right.
$$

It is elementary that this simultaneous equation has no solutions for $0<k<\pi / 6$. Thus total number of boundary preserving maps is equal to $8+4=12$.

Since there does not exist a 4-ply connected domain with conformal automorphism of order 6 , the maximal number of conformal self mappings of such a domain next to 8 is equal to 4 . Such a domain has a conformal representation as a circular slit annulus $1 / R<|z|<R(R>1)$ slit along two symmetric 
circular arcs on the unit circle. Clearly $f_{1}(z)=z^{2}$ and $f_{2}(z)=1 / z^{2}$ are boundary preserving maps of degree two. The image domain of the circular slit annulus under $f_{1}$ is triply connected and there exist at most six boundary preserving maps of degree two. For the domains with conformal automorphism of order less than 4 , the number of boundary preserving maps is at most 6 . Hence we obtain

THEOREM 3. The total number of boundary preserving maps of a 4-ply connected domain is at most 12 and $A_{4}=12$.

\section{REFERENCES}

[1] HeIns, H., On the number of 1-1 conformal maps which a multiply-connected plane region of finite connectivity $\mathrm{p}(>2)$ admits onto itself. Bull. Amer. Math. Soc. 52 (1946), 454-457.

[2] Howard, A. And A.J. Sommes, On the theorem of de Franchis Ann. Scoula Norm. Sup. Pisa 10 (1983), 429-436.

[3] Samuel, P., Lectures on old and new results on algebraic curves, Tata Institute of Fundamental Research, Bombay, 1966.

[4] Sario, L. ANd OiKawA, K., Capacity functions. Springer, Berlin-Heiderberg-New York (1970).

[5] Suita, N., Minimal slit domain and minimal sets. Kōdai Math. Sem. Rep. 17 (1965), 166-186.

Department of Mathematics

WASHINGTON UNIVERSITY

AND

Department of Mathematics

Tokyo Institute of Technology 